BULL. AUSTRAL. MATH, SOC.

VOL. 36 (1987) 515-520

\title{
A REMARK ON PROJECTIVE MODULES
}

\author{
WOJCIECH KUCHARZ
}

Let $R$ denote the field of real numbers and let $A$ be the ring of regular functions on $R^{n}$, that is the localization of $R\left[T_{1}, \ldots, T_{n}\right]$ with respect to the set of all polynomials vanishing nowhere on $R^{n}$. Let $X$ be an algebraic subset of $R^{n}$ and let $I(X)$ be the ideal of $A$ of all functions vanishing on $X$. Assume that $X$ is compact and nonsingular and $k=\operatorname{codim} X=1$, 2,4 or 8 . We prove here that if the $A / I(X)$-module $I(X) / I(X)^{2}$ can be generated by $k$ elements, then there exist a projective $A$-module $P$ of rank $k$ and a homomorphism from $P$ onto $I(X)$.

\section{Introduction}

Let $R$ denote the field of real numbers and let $A$ be the ring of all functions $f: R^{n} \rightarrow R$ such that $f=\phi / \psi$ for some polynomial functions $\phi, \psi: R^{n} \rightarrow R$ with $\psi$ vanishing nowhere. In other words, $A$ is (isomorphic tol the localization of the polynomial ring $R\left[T_{1}, \ldots, T_{n}\right]$ with respect to the set consisting of all polynomials vanishing nowhere on $R^{n}$. Given a subset $X$ of $R^{n}$, we denote by $I(X)$ the ideal of $A$ of all functions vanishing on $X$.

Received 24 February 1987.

Copyright Clearance Centre, Inc. Serial-fee code: 0004-9729/87 $\$ A 2.00+0.00$ 
In this note we prove the following.

THEOREM. Let $X$ be a nonsingular algebraic subset of $R^{n}$ of codimension $k$. Assume that the $A / I(X)$-mocule $I(X) / I(X)^{2}$ can be generated by $k$ ezements. If $k=1,2,4$ or 8 and $X$ is compact, then there exist a finitely generated projective A-module $P$ of rank $k$ and $a$ surjective homomorphism $h: P \rightarrow I(X)$.

For $k=1$ or 2 some better results are known. Indeed, since $A$ is a factorial ring (being a localization of $R\left[T_{1}, \ldots, T_{n}\right]$ ), the ideal $I(X)$ is principal if $k=1$, without the compactness assumption. If $k=2$, then the ideal $I(X)$ is a complete intersection (see [4]) and one can even drop the compactness assumption for $\operatorname{dim} X=1$ (see [8]). Moreover, the theorem holds true if $k=2$ but $X$ is not necessarily compact (see for example, [7, Theorem 3.1]).

It is unknown whether all finitely generated projective $A$-modules of rank greater than one are free (proofs of the serre conjecture concerning finitely generated projective modules over polynomial rings do not seem to extend to this case, see [10],[12]). Therefore the theorem does not allow us to conclude that the ideal $I(X)$ is a complete intersection for $k=2,4$ or 8 (see the remark above for $k=2$ ).

The author does not know whether the theorem remains true for $k=4$ or 8 if one drops the compactness assumption or replaces $R$ by another, say real closed, field.

\section{Proof of the Theorem}

our terminology and notions concerning real algebraic geometry are consistent with those of [2], [3] and [13]. In particular, $A$ is the ring of regular functions on $R^{n}$ (see [3, Chapter 3] or [11]). Also recall that an algebraic vector bundle $\xi$ over an affine real algebraic variety $X$ is said to be strongly algebraic if there exists an algebraic bundle $\eta$ over $X$ such that $\xi \otimes \eta$ is algebraically isomorphic to a product vector bundle $X \times R^{m}$ (see [2], [3, Chapter 12] and [13]).

EXAMPLE 1. The real projective space $R P^{n}$ with its standard structure of an abstract real algebraic variety is an affine variety (see [3, Theorem 3.4.4] or $[1, \mathrm{p} .432])$. Moreover, every $C^{\infty} R$-vector bundle 
over $R P^{n}$ is $C^{\infty}$ isomorphic to a strongly algebraic vector bundle (see [3, Example 12.3.7(c)]). Indeed, let $\xi$ be a $C^{\infty} R$-vector bundle over $R P^{n}$. Then $\xi$ is stably equivalent to the canonical line bundle $\gamma^{n}$ over $R P^{n}$ or to the direct sum of several copies of $\gamma^{n}[6, p .223$, Theorem 12.7]. Obviously, $\gamma^{n}$ is strongly algebraic and hence $\xi$ is stably equivalent to a strongly algebraic vector bundle. It follows that $\xi$ is $c^{\infty}$ isomorphic to a strongly algebraic vector bundle (see $[2, p$. 109]).

The next technical result is proved in [13, Proposition 2].

LEMMA 2. Let $X$ be an affine nonsingular real algebraic variety and let $\xi$ be a strongly algebraic vector bundle over $X$. Assume that $X$ is compact in the Euclidean topology. If $s$ is a $c^{\infty}$ section of $\xi$ vanishing on a closed nonsingular algebraic subvariety $Y$ of $X$, then there exists an algebraic section $u$ of $\xi$ which is arbitrarily close to $s$ in the $C^{\infty}$ topology and vanishes on $Y$.

The last auxiliary result is the following.

LEMMA 3. Let $A$ be a closed $C^{\infty}$ submanifold of a $C^{\infty}$ manifold $M$. Assume that the normal vector bundle of $A$ in $M$ is trivial. If $\operatorname{codim} A=1,2,4$ or 8 , then there exist a $C^{\infty}$-vector bundle $\xi$ over $M$ and $a C^{\infty}$ section $s$ of $\xi$ such that rank $\xi=\operatorname{codim} A, s$ is transverse to the zero section of $\xi$ and the set of zeros $s^{-1}(0)$ of $s$ is equal to $A$.

Proof. Let $k=\operatorname{codim} A$ and let $S^{k}$ be the unit $k$-dimensional sphere. Since the normal vector bundle of $A$ is trivial, there exist a $C^{\infty} \operatorname{map} f: M \rightarrow S^{k}$ and a regular value $y$ of $f$ such that $f^{-1}(y)=A$ (see [9]). If $k=1,2,4$ or 8 , then one can find a $C^{\infty} R$-vector bundle $\gamma$ over $S^{k}$ and a $C^{\infty}$ section $u$ of $\gamma$ such that rank $\gamma=k, u$ is transverse to the zero section of $\gamma$ and $u^{-1}(0)=\{y\}$ (the construction of $\gamma$ and $u$ is easily available if one identifies $s^{1}, s^{2}, s^{4}$ and $s^{8}$ with the projective line over the reals, complexes, quaternions and Cayley 
numbers, respectively). It suffices to set $\xi=f^{*} \gamma$ and $s=f^{*} u$, where, as usual, $f^{*} \gamma$ denotes the pull-back vector bundle and $f^{*} u$ denotes the pull-back section.

Proof of the Theorem. We identify $R^{n}$ with a subset of $R P^{n}$ via the map which sends $\left(x_{1}, \ldots, x_{n}\right)$ to $\left[1, x_{1}, \ldots, x_{n}\right]$. Let $Y$ be the Zariski closure of $X$ in $R P^{n}$. Then $Y=X \cup X^{\prime}$, where $X^{\prime}$ is contained in $R P^{n} \backslash R^{n}$. Notice that $X$ is a $C^{\infty}$ submanifold of $R P^{n}$ and the normal vector bundle of $X$ is trivial. It follows from Lemma 3 that there exist a $C^{\infty}$ vector bundle $\xi$ over $R P^{n}$ and a $C^{\infty}$ section $s$ of $\xi$ such that rank $\xi=k, s$ is transverse to the zero section of $\xi$ and $s^{-1}(0)=X$. By Example 1, we can assume that $\xi$ is a strongly algebraic vector bundle.

Let Sing $(Y)$ be the set of singular points of $Y$. By the Hironaka theorem [5], there exist a nonsingular xeal algebraic variety $V$ and a real algebraic morphism $\pi: V \rightarrow R P^{n}$ such that $\pi$ isomorphically transforms $V \backslash \pi^{-1}(\operatorname{sing}(Y))$ onto $R P^{n} \backslash \operatorname{sing}(Y)$ and the zariski closure $Z$ of $\pi^{-1}(Y \backslash \operatorname{sing}(Y))$ in $V$ is nonsingular. Moreover, since $\pi$ is the composition of finitely many algebraic blowing-ups, it is a proper map in the Euclidean topology (in particular, $V$ is compact) and $V$ is an affine real algebraic vareity. Notice that $Z=Z_{1} \cup Z_{2}$, where $Z_{1}=\pi^{-1}(X)$ and $z_{2}$ is a zariski closed subset of $V$ disjoint from $z_{1}$. since $Z$ and $Z_{2}$ are both Zariski closed, $Z$ is nonsingular and $\operatorname{dim} Z=\operatorname{dim} Z_{2}$, it follows that $Z_{1}$ is Zariski closed in $V$ (see [1, Lemma 1.6]) and, of course, nonsingular.

Clearly, the pull-back vector bundle $\pi^{*} \xi$ over $V$ is strongly algebraic and the pull-back section $\pi^{*} s$ is of class $C^{\infty}$ and transverse to the zero section of $\pi^{*} \xi$ and $\left(\pi^{*} s\right)^{-1}(0)=2_{1}$. By Lemma 2 , there exists an algebraic section $\nu$ of $\pi^{*} \xi$ arbitrarily close to $s$ in the $C^{\infty}$ topology and vanishing on $2_{1}$. Thus we can assume that $v$ is transverse to the zero section of $\pi^{*} \xi$ and $\nu^{-1}(0)=z_{1}$. 
Let $\eta$ be the restriction of $\xi$ to $R^{n}$ and let $\rho=\left(\pi \mid \pi^{-1}\left(R^{n}\right)\right)^{-1}$. Then $\eta=\rho^{*}\left(\pi^{*} \xi \mid \pi^{-1}\left(R^{n}\right)\right)$ and $u=\rho^{*}$ is an algebraic section of $\eta$ which is transverse to the zero section of $\eta$ and satisfies $X=u^{-1}(0)$.

Let $Q$ be the A-module of all algebraic sections of $\eta$. It follows from the definition of a strongly algebraic vector bundle that $Q$ is a finitely generated projective module of rank $k$ (see also [3, Proposition 12.1.11]) and hence so is the module $P=\operatorname{Hom}(Q, A)$. Since $u$ is transverse to the zero section of $n$ and $u^{-1}(0)=X$, one easily sees that for every $\alpha$ in $P$, the element $\alpha(U)$ belongs to $I(X)$ and all elements of this form generate $I(X)$. To conclude the proof, we define $h: P \rightarrow I(X)$ by $h(\alpha)=\alpha(u)$ for $\alpha$ in $P$.

\section{References}

[1] S. Akbulut and H. King, "The topology of real algebraic sets with isolated singularities," Ann. of Math. 113,(1981), 425-446.

[2] R. Benedetti and A. Tognoli, "On real algebraic vector bundles," Buzl. Sci. Math. (2) 104 (1980), 89-112.

[3] J. Bochnak, M. Coste and M.F. Roy, Géométrie Algébrique Réelle, (Ergebnisse der Math. Vol. 12, Springer 1987).

[4] J. Bochnak and W. Kucharz, "Real algebraic surfaces as complete intersections," BuZZ. London Math. Soc. 19 (1987), 149-153.

[5] H. Hironaka, "Resolution of singularities of an algebraic variety over a field of characteristic zero," Ann. of Math. 79 (1964), 109-326.

[6] D. Husemoller, Fibre Bundles, (Graduate Texts in Mathematics 20, second edition, Springer 1975), 209-225.

[7] W. Kucharz, "Vector bundles over real algebraic surfaces and threefolds," Compositio Math 60 (1986), 209-225.

[8] W. Kucharz, "Real algebraic curves as complete intersections," Math. Z., 194 (1987), 259-266.

[9] J. Milnor, Topology from the Differentiable Viewpoint, (Univ. of Virginia Press, Charlottesville 1966).

[10] D. Quillen, "Projective modules over polynomial rings," Invent. Math. 36 (1976), 167-171. 
[11] R. Silhol, "Géométrie algébrique sur un corps non algébriquement clos," Comm. Algebra 6 (1978), 1134-1165.

[12] A. Suslin, "Projective modules over polynomial rings," (Russian), DokZ. Akad. Nauk. S.S.S.R. 229 (1976), 1063-1066.

[13] A. Tognoli, "Algebraic approximation of manifolds and spaces," Séminair Bourbaki vol. 1979/80 (Springer Lect. Notes in Math. $842,73-94,1981)$.

Department of Mathematics and Statistics

University of New Mexico

Albuquerque, New Mexico 87131

U.S.A. 